\title{
探讨民商法视角下谈如何保护公民私有财产
}

\author{
钟海华 \\ 筕州广播电视大学 \\ DOI:10.32629/ej.v2i6.315
}

[ 摘 要] 随着社会在不断向前发展,公民私有财产的保护对于社会发展、法治社会的构建与完善有着非常重要的意义。目前,民商法对于公民 私有财产保护过程中,仍然有很多不足需要不断的完善。本文主要通过解析现阶段民商法在保护公民私有财产存在的问题同时,提出一系列相应 的改善对策。

[关键词] 民商法; 私有财产; 保护

随着社会的发展, 公民私有财产的地位也不断在提升, 保护公民的私 有财产是国家进步的重要标志。我国现有保护公民私有财产的法律法规较 多, 像刑法、民商法、物权法、经济法等都有对保护财产进行规定, 在诸多 法律法规中, 民商法占据主要地位, 它是对公民私有财产保护最为直接的 法律体系。由于一系列的原因, 目前我国民商法在保护公民私有财产时还 有一系列问题, 这对法律的权威产生了不好的影响, 需要在司法实践中逐 一进行解决。

\section{1 民商法在公民私有财产保护中的不足}

民商法与公民的工作与生活息息相关, 它为保护公民的私有财产提供 了坚实有力的法律保障, 但是民商法在公民私有财产保护时仍有很多的不 足, 需要特别注意。

\section{1 公民私有财产产权意识较为薄弱}

受到我国长期计划经济思想体制的严重影响, 很多公民对于私有财产 的保护意识还不太强烈, 没有积极主动的来利用民商法保护自己的私有财 产。有些非公有制企业主和公民在私人财产遇到非法侵害时, 首先想到的 不是如何运用法律的武器来武装自己, 而是选择被动接受, 更为极端的就 是采取非法暴力的手段自行解决, 这样以来没有得到应有的赔偿, 反而造 成触犯法律。另外, 很多公民缺乏对民法、商法的性质、条款内容细致的 了解, 没有使民商法保护私有财产方面受到应有的重视。

1. 2 私有财产监管制度监管不严格

在保护公民私有财产不受侵犯过程中, 民商法坚持的基本原则就是 “不主动干预” , 在私有财产遭受到侵害行为时, 才需要做出相应的措施。 现阶段, 我国法律体系中, 没有专门性的组织、机构来监管保护公民的私有 财产, 所以在处理私有财产矛盾或财产纠纷时, 尤其是在私有财产维权过 程中, 由于监管不严格, 很容易出现腐败的现象, 这就严重影响到对公民私 有财产的保护, 对于民商法的权威性与可信度造成了恶劣的影响。

1.3 实际生活中缺乏对民商法的应用

不论哪一部法律, 如果没有落实到现实生活中去, 那么将会变成一纸 空文, 没有真正的将它转变成现实权利。国家从公民的切身利益出发, 制定 出的任何法律目的就是为了切实保护每位公民的自身利益, 使公民真正享 受这项法律赋予的权利。目前来看, 民商法还没有真正的将保护公民私有 财产充分落实到现实生活中去。

\section{2 民商法视角下保护公民私有财产的策略}

2. 1 强化法律普及宣传, 提高公民私有财产产权意识

目前, 我国公民普遍存在对民商法认识不足, 私有财产权意识薄弱的
情况, 针对这些问题点, 应该加快科普宣传工作, 可以借助电视、广播、宣 传栏等传统媒体, 也可以通过微信、微博、抖音视频等新型网络媒体方式进 行。与此同时, 要加强对民商法宣传工作的监管, 确认宣传工作是否真正落 实, 建立健全普法宣传体制, 让更多的公民能够认识到民商法在保护公民私 有财产时的价值与发挥的作用, 并提倡他们学习民商法的相关条款, 使他们 在私有财产受到侵害时主动拿起法律的武器保护自己的财产不受损害。

2. 2 构建法律监管体系

现在的社会, 经济高速发展, 互联网的影子无处不在, 若想真正实现保 护公民的私有财产, 必须有健全的法律监管体系, 并且能够严格执行, 严厉 打击司法腐败的行为, 真正意义上实现民商法有效保护公民的私有财产。 因此, 在实际构建法律监管体系时, 必须以下几点: 首先单独设立法律监管 部门, 该部门主要职责是处理与解决涉及私有财产方面的纠纷与矛盾; 其 次在执法机构所涉及的关联岗位职能要清晰、明了, 执法过程中, 如果发现 触犯民商法行为的案件, 及时追究责任, 并进行依法处置, 维护民商法的公 平、公正, 维护法律的权威。

2.3 充分落实民商法的司法实践

落实民商法的司法实践是保护公民私有财产不受侵犯的最低要求, 对 每一位公民享受法律赋予权利具有重要的意义。司法实践只有落实到了实 处, 才能够强有效的保证每一个公民行使权力, 使他们从心底真切的产生 对法律的敬意。落实民商法的司法实践不仅帮助公民实现财产的保护, 还 可以帮助我国建立全面的司法制度, 进而引导公民对法律产生崇敬之情。

\section{3 结束语}

随着我国改革开放的不断深入, 我国以公有制为主题, 多种所有制共 存的经济体制已经形成, 公民私有财产也出现了较大的变化, 对于私有财 产的保护也迫在眉睫, 显得更加重要。目前我国民商法在保护给公民私有 财产的同时, 还存在诸多的问题需要解决, 需要不断提高公民的依法维权 意识, 强化监督部门的监管力度, 充分落实司法实践, 使民商法保护公民私 有财产方面发挥出重要的作用, 进而促进我国公民收入的提高。

\section{[参考文献]}

[1]田阳.基于民商法视角下谈如何保护公民私有财产 [J].法制博 览,2017(4):251.

[2]王梓睿,郭晓霜.关于私有财产的民商法保护剖析[J].科技展 望,2016(24):323.

[3] 盛钧.私有财产的民商法保护原则析述[ J]. 湖南税务高等专科学校 学报,2015(4):45-46+55. 